\title{
RATIONAL MAPS ADMITTING MEROMORPHIC INVARIANT LINE FIELDS
}

\author{
XIAOGUANG WANG
}

\author{
(Received 23 December 2008)
}

\begin{abstract}
It is shown that a rational map of degree at least 2 admits a meromorphic invariant line field if and only if it is conformally conjugate to either an integral Lattès map, a power map, or a Chebyshev polynomial.

2000 Mathematics subject classification: primary 37F10; secondary 37F15.

Keywords and phrases: invariant line field, Lattès map, Chebyshev polynomial.
\end{abstract}

\section{Introduction}

A line field supported on a subset $E$ of the complex sphere $\overline{\mathbb{C}}$ is the Beltrami differential $\mu=\mu(z) d \bar{z} / d z$ supported on $E$ with $|\mu|=1$. We say $\mu$ is measurable if $\mu(z)$ is a measurable function. Let $f$ be a rational map of degree $\operatorname{deg}(f) \geq 2$. We say $f$ admits an invariant line field if there is a measurable line field $\mu$ supported on a set in $\overline{\mathbb{C}}$ with positive measure such that $f^{*} \mu=\mu$ almost everywhere (refer to [3]).

We are mostly interested in the invariant line fields which are carried on the Julia sets for rational maps. One well-known example is the so called 'integral Lattès map', which is constructed via a torus endomorphism. The construction is as follows. Let $X=\mathbb{C} / \Lambda$ be a complex torus and let $\alpha$ be a complex number such that $|\alpha|>1$ and $\alpha \Lambda \subset \Lambda$. Multiplication by $\alpha$ induces an endomorphism $F: X \rightarrow X$. Let $\wp: X \rightarrow \overline{\mathbb{C}}$ be the Weierstrass function. Since $\wp(-z)=\wp(z)$, the endomorphism $F$ induces a rational map $f: \overline{\mathbb{C}} \rightarrow \overline{\mathbb{C}}$ such that $f(\wp(z))=\wp(F(z))$. Such a map $f$ is called a Lattès map. If $\alpha$ is an integer, then $F$ admits an invariant line field on $X$. This line field has the form $e^{i \theta} d \bar{z} / d z$ and can descend to an invariant line field for $f$ (see [3]). On the other hand, we can verify that if $f$ admits an invariant line field, then this line field lifts to an invariant line field for $F$ and $\alpha$ turns out to be an integer. In this case, we say $f$ is an integral Lattès map.

One of the central problems in complex dynamics is the following.

The research was supported by the National Natural Science Foundation of China Grant 10871047.

(C) 2009 Australian Mathematical Publishing Association Inc. 0004-9727/2009 \$16.00 
Conjecture 1.1 (No invariant line fields). A rational map $f$ of degree $\operatorname{deg}(f) \geq 2$ carries no invariant line fields on its Julia set, except when $f$ is an integral Lattès map.

The conjecture implies the density of hyperbolic maps in the space Rat ${ }_{d}$ of all rational maps of degree $d$ (see [3]). Much study has been devoted to special families of rational maps, especially quadratic polynomials of the form $f_{c}(z)=z^{2}+c$ for $c \in \mathbb{C}$. However, even for the quadratic family, the conjecture is still unsolved.

Fortunately, if we require the line field $\mu$ to be 'good', we can classify all the rational maps which leave $\mu$ invariant. Here, a 'good' line field means that it can be written in the form $\mu=\bar{\phi} /|\phi|$, where $\phi$ is a nonzero meromorphic quadratic differential defined on $\overline{\mathbb{C}}$. In this case, formally, we call $\mu$ a meromorphic line field, dual to $\phi$. Correspondingly, we say that $f$ admits a meromorphic invariant line field if $f^{*} \mu=\mu$.

Now we can formulate our main theorem.

THEOREM 1.2. Let $f$ be a rational map of degree $\operatorname{deg}(f) \geq 2$. Then $f$ admits a meromorphic invariant line field if and only if $f$ is conformally conjugate to one of the following maps.

(1) an integral Lattès map;

(2) the power map $z \mapsto z^{d}$, for $d \in \mathbb{Z}$ and $|d| \geq 2$;

(3) $\pm T_{n}, n \geq 2$, where $T_{n}$ is the $n t h$ Chebyshev polynomial defined by $T_{n}(2 \cos z)=$ $2 \cos (n z)$.

This theorem is deeply inspired by a theorem in [3] which states that if a rational map $f$ admits an invariant line field which is holomorphic on a nonempty open set contained in the Julia set, then $f$ is an integral Lattès map. Moreover, three examples are provided in [3]. One is the power map $z \mapsto z^{d}$, for which the line field is dual to $d z^{2} / z^{2}$; another is the integral Lattès map, for which the line field is dual to

$$
\frac{d z^{2}}{\left(z-p_{1}\right)\left(z-p_{2}\right)\left(z-p_{3}\right)\left(z-p_{4}\right)}
$$

the third is the quadratic polynomial $f(z)=z^{2}-2$, for which the line field is dual to $d z^{2} /\left(z^{2}-4\right)$. So it is a natural question to determine whether these are all examples which admit meromorphic invariant line fields. These examples motivate our study.

It is interesting to compare our classification theorem with another trichotomy theorem from the viewpoint of 'permutable maps'. Motivated by [5], we call a rational map $f$ permutable if it commutes with another rational map $g, f \circ g=g \circ f$, where both $f$ and $g$ have degree at least 2, and no iterate of $f$ is equal to an iterate of $g$.

THEOREM 1.3 (Ritt [6] and Eremenko [2]). A rational map $f$ of degree $\operatorname{deg}(f) \geq 2$ is permutable if and only if it is a finite quotient of an affine map; that is, if and only if it is either a Lattès, Chebyshev, or power map.

This theorem was first proved by Ritt [6] in 1923, and by Eremenko [2] using a quite different method in 1989. For higher-dimensional analogues, see [1]. 


\section{Proof of the main theorem}

First we need some notation. Let $\mathcal{M}(\overline{\mathbb{C}})$ be the set of all meromorphic quadratic differentials defined on $\overline{\mathbb{C}}$. For $\phi \in \mathcal{M}(\overline{\mathbb{C}})$, let $\mathcal{Z}(\phi)$ and $\mathcal{P}(\phi)$ be the zero set and the pole set of $\phi$, respectively. The order of $\phi \in \mathcal{M}(\overline{\mathbb{C}})$ at a point $z_{0}$, denoted by $\operatorname{ord}_{z_{0}}(\phi)$, is defined as follows. If $z_{0}$ is a zero of $\phi$ of order $n$, set $\operatorname{ord}_{z_{0}}(\phi)=n$; if $z_{0}$ is a pole of $\phi$ of order $n$, set $\operatorname{ord}_{z_{0}}(\phi)=-n$; for other cases, where $z_{0}$ is called a regular point of $\phi$, set $\operatorname{ord}_{z_{0}}(\phi)=0$. For a rational map $f$, let $C(f)$ be the set of all critical points, and let

$$
P(f)=\bigcup_{c \in C(f), n>0} f^{n}(c)
$$

be the postcritical set. The backward orbit of a point $z$, under iteration of $f$, is denoted by $\operatorname{orb}^{-}(z)=\bigcup_{n \geq 0} f^{-n}(z)$. Let $\operatorname{deg}(f, z)$ be the local degree of $f$ at $z$.

Proof of TheOrem 1.2. Let $f$ be a rational map of degree $\operatorname{deg}(f) \geq 2$ and $\mu=$ $\bar{\phi} /|\phi|$ be a meromorphic invariant line field of $f$ for some $\phi \in \mathcal{M}(\overline{\mathbb{C}})$.

The 'if' part of the theorem is easy to verify. The proof for the 'only if' part consists of five steps.

STEP 1. $f^{*}(\bar{\phi} /|\phi|)=\bar{\phi} /|\phi|$ if and only if there is a positive constant $C$ such that $f^{*} \phi=C \phi$. This constant $C$ is uniquely determined by $f$. Moreover, any other meromorphic invariant line field of $f$ must have the form $e^{i \theta} \mu$ for some $\theta \in \mathbb{R}$.

Note that the relation $f^{*}(\bar{\phi} /|\phi|)=\bar{\phi} /|\phi|$ is equivalent to

$$
f^{*} \phi / \phi=\left|f^{*} \phi\right| /|\phi| \text {. }
$$

This indicates that the well-defined holomorphic map $f^{*} \phi / \phi: \overline{\mathbb{C}} \rightarrow \overline{\mathbb{C}}$ takes only positive values, thus equation (2.1) holds if and only if $f^{*} \phi / \phi$ is a positive constant by open map theorem.

Now suppose $\mu_{i}=\bar{\phi}_{i} /\left|\phi_{i}\right|(i=1,2)$ are two meromorphic invariant line fields for $f$. The above argument shows $f^{*} \phi_{i}=C_{i} \phi_{i}, i=1,2$. Since $\phi_{1} / \phi_{2}$ is a welldefined holomorphic map from $\overline{\mathbb{C}}$ to itself, denoted by $R$, the relation

$$
\frac{f^{*} \phi_{1}}{f^{*} \phi_{2}}=\frac{C_{1} \phi_{1}}{C_{2} \phi_{2}}
$$

implies that $R \circ f=\left(C_{1} / C_{2}\right) R$. Comparing the degree of $R \circ f$ and $f$, we conclude that $R$ is a nonzero complex constant, and $C_{1}=C_{2}$. Therefore $\mu_{1}$ is identical to $\mu_{2}$ up to a rotation.

From now on, we may write $\phi$ and $C$ as $\phi_{f}$ and $C_{f}$ since they are determined by $f$. To find all rational maps which admit meromorphic invariant line fields is equivalent to finding all solutions $\left(f, \phi_{f}, C_{f}\right) \in \mathrm{Rat}_{2}^{+} \times \mathcal{M}(\overline{\mathbb{C}}) \times \mathbb{R}^{+}$to the indeterminate equation

$$
f^{*} \phi_{f}=C_{f} \phi_{f}
$$


where $\mathrm{Rat}_{2}^{+}$is the space of all rational maps of degree at least 2. In local coordinates, $\phi_{f}=\phi_{f}(z) d z^{2}$, the indeterminate equation has the form

$$
\phi_{f}(f(z)) f^{\prime}(z)^{2}=C_{f} \phi_{f}(z)
$$

Moreover, for any $z \in \overline{\mathbb{C}}$, comparing the order of $f^{*} \phi_{f}$ and $\phi_{f}$ at the point $z$, we have the identity

$$
\operatorname{ord}_{z}\left(f^{*} \phi_{f}\right)=\operatorname{deg}(f, z)\left(2+\operatorname{ord}_{f(z)}\left(\phi_{f}\right)\right)-2=\operatorname{ord}_{z}\left(\phi_{f}\right) .
$$

STEP 2. $\mathcal{Z}\left(\phi_{f}\right)=\emptyset$.

Otherwise let $z_{0} \in \mathcal{Z}\left(\phi_{f}\right) \neq \emptyset$. We can conclude from equation (2.2) that orb $^{-}\left(z_{0}\right) \subset \mathcal{Z}\left(\phi_{f}\right)$. Since $\mathcal{Z}\left(\phi_{f}\right)$ is a discrete subset of $\overline{\mathbb{C}}$, \# orb ${ }^{-}\left(z_{0}\right)<\infty$ and $\# f^{-1}\left(\operatorname{orb}^{-}\left(z_{0}\right)\right) \geq \# \operatorname{orb}^{-}\left(z_{0}\right)$. On the other hand,

$$
f^{-1}\left(\operatorname{orb}^{-}\left(z_{0}\right)\right)=\bigcup_{n \geq 1} f^{-n}\left(z_{0}\right) \subset \operatorname{orb}^{-}\left(z_{0}\right) .
$$

So we have $f^{-1}\left(\operatorname{orb}^{-}\left(z_{0}\right)\right)=\operatorname{orb}^{-}\left(z_{0}\right)$. It is easy to see that all points in $\operatorname{orb}^{-}\left(z_{0}\right)$ are superattracting periodic points. If $\# \operatorname{orb}^{-}\left(z_{0}\right) \geq 3$, by Montel's theorem, the set $\overline{\mathbb{C}} \backslash$ orb $^{-}\left(z_{0}\right)$ is completely invariant and lies in the Fatou set $F(f)$. This indicates that the Julia set $J(f)=\emptyset$, which is a contradiction. Thus \# orb $^{-}\left(z_{0}\right)$ can only be 1 or 2 .

If \# orb ${ }^{-}\left(z_{0}\right)=2$, then $f$ is conformally conjugate to the power map $z \mapsto z^{d}$, for some $d \in \mathbb{Z}$. But it is known that any meromorphic invariant line field of the power map must be dual to $C d z^{2} / z^{2}$ (see Section 1 and Step 1), which has no zeros. So this case is impossible.

If \# $\operatorname{orb}^{-}\left(z_{0}\right)=1$, then $f^{-1}\left(z_{0}\right)=\left\{z_{0}\right\}$, and $\operatorname{deg}\left(f, z_{0}\right)=\operatorname{deg}(f)$. By identity (2.3), we have

$$
\operatorname{ord}_{z_{0}}\left(f^{*} \phi_{f}\right)=\operatorname{deg}(f)\left(\operatorname{ord}_{z_{0}}\left(\phi_{f}\right)+2\right)-2=\operatorname{ord}_{z_{0}}\left(\phi_{f}\right) .
$$

But this is also impossible since $\operatorname{ord}_{z_{0}}\left(\phi_{f}\right) \geq 1, \operatorname{deg}(f) \geq 2$.

STEP 3. $f$ is critically finite, that is $\# P(f)<\infty$. Moreover, $P(f)=\mathcal{P}\left(\phi_{f}\right)$.

For any $c \in C(f)$, equation (2.2) implies that $f(c) \in \mathcal{P}\left(\phi_{f}\right)$. Otherwise $c$ will be a zero of $\phi_{f}$, which is already ruled out in Step 2. Replacing $f$ by $f^{n}$, we have $f^{n}(c) \in \mathcal{P}\left(\phi_{f}\right)$, thus

$$
\bigcup_{n \geq 0} f^{n}(C(f)) \subset \mathcal{P}\left(\phi_{f}\right) .
$$

This means $f$ is critically finite, since $\mathcal{P}\left(\phi_{f}\right)$ is a finite set. Moreover, $P(f) \subset \mathcal{P}\left(\phi_{f}\right)$.

If $\mathcal{P}\left(\phi_{f}\right) \backslash P(f) \neq \emptyset$, taking $z_{0} \in \mathcal{P}\left(\phi_{f}\right) \backslash P(f)$, we have from equation (2.2) that

$$
\operatorname{orb}^{-}\left(z_{0}\right) \subset \mathcal{P}\left(\phi_{f}\right), \quad \# \operatorname{orb}^{-}\left(z_{0}\right)=\infty,
$$

which is a contradiction. This ends the proof of Step 3. 
By the Riemann-Roch theorem,

$$
\operatorname{deg}\left(\phi_{f}\right)=\# \mathcal{Z}\left(\phi_{f}\right)-\# \mathcal{P}\left(\phi_{f}\right)=-\# \mathcal{P}\left(\phi_{f}\right)=-4 .
$$

This means that $\phi_{f}$ has four poles (counting the multiplicity). Since $f$ is critically finite, each periodic cycle of $f$ is either repelling or superattracting (see [3] or [4]).

STEP 4. If $f$ has no superattracting cycle, then $f$ is an integral Lattès map.

The proof in this step is due to McMullen (compare [3]). For completeness, we include it here. If $f$ has no superattracting cycle, then the Julia set $J(f)=\overline{\mathbb{C}}$.

We first show that $\phi_{f}$ has four simple poles. That is, up to a constant,

$$
\phi_{f}=\frac{d z^{2}}{\left(z-p_{1}\right)\left(z-p_{2}\right)\left(z-p_{3}\right)\left(z-p_{4}\right)} .
$$

Indeed, if $\phi_{f}$ has a pole $p_{0}$ of order 2 or more, that is, $\operatorname{ord}_{p_{0}}\left(\phi_{f}\right) \leq-2$, then we can conclude from identity (2.3) by induction that for any $z \in \operatorname{orb}^{-}\left(p_{0}\right), \operatorname{ord}_{z}\left(\phi_{f}\right) \leq$ -2 , therefore $\operatorname{orb}^{-}\left(p_{0}\right) \subset \mathcal{P}\left(\phi_{f}\right)$. An argument similar to Step 2 indicates that $f^{-1}\left(\operatorname{orb}^{-}\left(p_{0}\right)\right)=\operatorname{orb}^{-}\left(p_{0}\right)$ and $\# \operatorname{orb}^{-}\left(p_{0}\right)=1$ or 2 . It turns out that $f$ is either conjugate to a power map or conjugate to a polynomial. But this will contradict $J(f)=\overline{\mathbb{C}}$.

Now we consider the orbifold $\mathcal{O}_{f}$ of $f$. Recall that the orbifold $\mathcal{O}_{f}$ of the critically finite map $f$ is a pair $\left(\overline{\mathbb{C}}, N_{f}\right)$, where $N_{f}: \overline{\mathbb{C}} \rightarrow \mathbb{N} \cup\{\infty\}$ takes values greater than 1 only on a discrete set of $\overline{\mathbb{C}}$. It is defined as follows:

(a) $\quad N_{f}(x)=1$, when $x \in \overline{\mathbb{C}} \backslash P(f)$;

(b) $\quad N_{f}(x)$ is the least common multiple of the local degrees in the set

$$
\left\{\operatorname{deg}\left(f^{n}, y\right) \mid f^{n}(y)=x, n \geq 1\right\}
$$

for $x \in P(f)$;

(c) $\quad N_{f}(x)=\infty$ if the local degrees in the set $\left\{\operatorname{deg}\left(f^{n}, y\right) \mid f^{n}(y)=x, n \geq 1\right\}$ are unbounded.

We claim that $\mathcal{O}_{f}=(\overline{\mathbb{C}},(2,2,2,2))$. In fact, it is easy to see that $N_{f}(x)=1$ when $x \in \overline{\mathbb{C}} \backslash P(f)$. For $x \in P(f)$, it is obvious that $N_{f}(x) \geq 2$. Let $z \in f^{-n}(x)$ for $n \geq 1$. Note that $\left(f^{n}\right)^{*} \phi_{f}=C_{f}^{n} \phi_{f}$; by identity (2.3),

$$
\operatorname{ord}_{z}\left(\left(f^{n}\right)^{*} \phi_{f}\right)=\operatorname{deg}\left(f^{n}, z\right)\left(2+\operatorname{ord}_{x}\left(\phi_{f}\right)\right)-2=\operatorname{ord}_{z}\left(\phi_{f}\right) .
$$

Since $P(f)=\mathcal{P}\left(\phi_{f}\right)$ and every pole of $\phi_{f}$ is simple in this case, $\operatorname{ord}_{x}\left(\phi_{f}\right)=-1$. Therefore $\operatorname{ord}_{z}\left(\phi_{f}\right)$ has only two choices 0 or -1 , and $\operatorname{deg}\left(f^{n}, z\right)$ can only be 1 or 2 . Thus $N_{f}(x) \leq 2$. The above argument shows that for any $x \in P(f), N_{f}(x)=2$. This proves the claim.

By [3, Theorem A.5], if $\mathcal{O}_{f}=(\overline{\mathbb{C}},(2,2,2,2))$, then $f$ is a Lattès map. By assumption, $f$ admits a meromorphic invariant line field, so $f$ is an integral 
Lattès map. Moreover, since $\phi_{f}$ is integrable over $\overline{\mathbb{C}}$, we have $C_{f}=\operatorname{deg}(f)$ from the identity

$$
\int_{\overline{\mathbb{C}}}\left|f^{*} \phi_{f}\right|=\operatorname{deg}(f) \int_{\overline{\mathbb{C}}}\left|\phi_{f}\right| .
$$

STEP 5. If $f$ has a superattracting cycle, then $f$ is either conjugate to a power map or conjugate to a Chebyshev polynomial.

Let $z_{0}$ be a superattracting point of $f$ with period $p$. From identity (2.3),

$$
\operatorname{ord}_{z_{0}}\left(\left(f^{p}\right)^{*} \phi_{f}\right)=\operatorname{deg}\left(f^{p}, z_{0}\right)\left(2+\operatorname{ord}_{z_{0}}\left(\phi_{f}\right)\right)-2=\operatorname{ord}_{z_{0}}\left(\phi_{f}\right) .
$$

Since $\operatorname{deg}\left(f^{p}, z_{0}\right) \geq 2$, we have $\operatorname{ord}_{z_{0}}\left(\phi_{f}\right)=-2$. Thus $z_{0}$ is a pole of $\phi_{f}$ of order 2 . Moreover, by identity (2.3) and induction, all preimages of $z_{0}$ are poles of $\phi_{f}$ of order 2. There are two possibilities:

(P1) $z_{0}$ is a fixed point of $f$ and $f^{-1}\left(z_{0}\right)=\left\{z_{0}\right\}$;

(P2) $z_{0}$ is of period 2 and $f^{-1}\left(z_{0}\right)=\{\zeta\}, f^{-1}(\zeta)=\left\{z_{0}\right\}$.

For (P1), there are two choices for $\phi_{f}$ up to a constant.

CASE 1. $\phi_{f}=\frac{d z^{2}}{\left(z-z_{0}\right)^{2}\left(z-z_{1}\right)^{2}}$.

CASE 2. $\phi_{f}=\frac{d z^{2}}{\left(z-z_{0}\right)^{2}\left(z-z_{1}\right)\left(z-z_{2}\right)}$.

In Case 1 , take $\gamma \in \operatorname{Aut}(\overline{\mathbb{C}})$, the automorphism group of $\overline{\mathbb{C}}$, such that $\gamma(0)=$ $z_{1}, \gamma(\infty)=z_{0}$. Then $\gamma^{*} \phi_{f}=C d z^{2} / z^{2}$ for some constant $C$ and $F=\gamma^{-1} \circ f \circ \gamma$ is a polynomial such that $F^{*}\left(\gamma^{*} \phi_{f}\right)=C_{f} \gamma^{*} \phi_{f}$. By conjugation, we may assume $f$ is a polynomial and $\phi_{f}=d z^{2} / z^{2}$. The equation $f^{*} \phi_{f}=C_{f} \phi_{f}$ is equivalent to

$$
\left(f^{\prime}(z) / f(z)\right)^{2}=C_{f} / z^{2} .
$$

Comparing the leading coefficients on both sides of (2.4), we have $C_{f}=\operatorname{deg}(f)^{2}$. It is easy to find the general polynomial solution $f(z)=A z^{d}$, where $A$ is a nonzero complex constant and $d=\operatorname{deg}(f)$. In this case, $f$ is conjugate to a power map.

In Case 2, take $\gamma \in \operatorname{Aut}(\overline{\mathbb{C}})$, such that $\gamma(\infty)=z_{0}, \gamma(-2)=z_{1}, \gamma(2)=z_{2}$. It is easy to show that $\gamma^{*} \phi_{f}=C d z^{2} /\left(z^{2}-4\right)$ for some constant $C$ and $F=\gamma^{-1} \circ f \circ \gamma$ is a polynomial. Thus as in Case 1 we assume that $f$ is a polynomial and $\phi_{f}=$ $d z^{2} /\left(z^{2}-4\right)$. The equation $f^{*} \phi_{f}=C_{f} \phi_{f}$ is equivalent to

$$
f^{\prime}(z)^{2}\left(z^{2}-4\right)=C_{f}\left(f(z)^{2}-4\right) .
$$

We want to find all polynomial solutions to this equation. First note that $f(2)=2$ or -2 if we set $z=2$. Comparing the leading coefficients on both sides, we have $C_{f}=\operatorname{deg}(f)^{2}$. To solve equation (2.5), we need a little trick. Let

$$
z=w+\frac{1}{w}, \quad f(z)=\varphi(w)+\frac{1}{\varphi(w)},
$$


where $\varphi(w)$ is required to be a holomorphic map; it need only be defined in some open set $U$ in $\mathbb{C}$. Indeed, since the map $w \mapsto w+w^{-1}$ is locally injective when $|w|$ is large, we can always do this. Calculation shows that

$$
f^{\prime}(z)=w \frac{\varphi^{\prime}(w)}{\varphi(w)} \frac{\varphi(w)-\varphi(w)^{-1}}{w-w^{-1}} .
$$

Then equation (2.5) becomes

$$
w \frac{\varphi^{\prime}(w)}{\varphi(w)}= \pm n, \quad w \in U,
$$

where $n=\operatorname{deg}(f)$. This new equation has general solution $\varphi(w)=C w^{n}$ or $\varphi(w)=$ $\left(C w^{n}\right)^{-1}$ for some indeterminate constant $C$, so

$$
f\left(w+\frac{1}{w}\right)=C w^{n}+\frac{1}{C w^{n}}, \quad w \in U .
$$

This relation in fact holds for all $w \in \overline{\mathbb{C}}$ by the identity theorem of holomorphic maps. If $f(2)=2$, then $C=1$. In this case $f(2 \cos z)=2 \cos (n z)$ if we write $w=e^{i z}$, and so $f=T_{n}$. If $f(2)=-2$, then $C=-1$. In this case $f=-T_{n}$. Therefore, in Case 2, $f$ is conjugate to a Chebyshev polynomial $T_{n}$ or $-T_{n}$.

The only remaining case is $(\mathrm{P} 2)$. In this case, we can easily show that $C_{f}=$ $\operatorname{deg}(f)^{2}, \phi_{f}=d z^{2} /\left(\left(z-z_{0}\right)^{2}(z-\zeta)^{2}\right)$ and $f$ is conjugate to the power map $z \mapsto z^{d}$, for $d \in \mathbb{Z}$ and $d \leq-2$. We omit the details here.

The proof is complete.

REMARK 2.1. For convenience we list all solutions to the indeterminate equation $f^{*} \phi_{f}=C_{f} \phi_{f}$ in the following table.

\begin{tabular}{cccc}
\hline$f$ is conjugate to & $\phi_{f}$ & $C_{f}$ & $\mathcal{O}_{f}$ \\
\hline Integral Lattès map & $\frac{d z^{2}}{\left(z-p_{1}\right)\left(z-p_{2}\right)\left(z-p_{3}\right)\left(z-p_{4}\right)}$ & $\operatorname{deg}(f)$ & $(\overline{\mathbb{C}},(2,2,2,2))$ \\
Power map & $\frac{d z^{2}}{\left(z-z_{0}\right)^{2}\left(z-z_{1}\right)^{2}}$ & $\operatorname{deg}(f)^{2}$ & $(\overline{\mathbb{C}},(\infty, \infty))$ \\
\pm Chebyshev polynomial & $\frac{d z^{2}}{\left(z-z_{0}\right)^{2}\left(z-z_{1}\right)\left(z-z_{2}\right)}$ & $\operatorname{deg}(f)^{2}$ & $(\overline{\mathbb{C}},(2,2, \infty))$ \\
\hline
\end{tabular}

We can see that for all cases $\mathcal{O}_{f}$ is a parabolic orbifold and $\sqrt{\left|\phi_{f}\right|}$ is an orbifold metric on $\mathcal{O}_{f}$.

\section{Acknowledgements}

The author would like to thank Weiyuan Qiu and Yongcheng Yin for their helpful comments and constant encouragement. 


\section{References}

[1] T. C. Dinh and N. Sibony, 'Sur les endomorphismes holomorphes permutables', Math. Ann. 324 (2002), 33-70.

[2] A. Eremenko, 'Some functional equations connected with iteration of rational functions', Algebra Anal. 1 (1989), 102-116 (in Russian). Leningrad Math. J. 1 (1990), 905-919 (in English).

[3] C. McMullen, Complex Dynamics and Renormalization, Annals of Mathematical Studies, 135 (Princeton University Press, Princeton, NJ, 1994).

[4] J. Milnor, Dynamics in One Complex Variable, 3rd edn, Annals of Mathematical Studies, 160 (Princeton University Press, Princeton, NJ, 2006).

[5] _ - 'On Lattès maps', in: Dynamics on the Riemann Sphere: A Bodil Branner Festschrift (eds. P. G. Hjorth and C. L. Peterson) (European Mathematical Society, 2006), pp. 9-43.

[6] J. F. Ritt, 'Permutable rational functions', Trans. Amer. Math. Soc. 25 (1923), 398-448.

XIAOGUANG WANG, School of Mathematical Sciences, Fudan University, Shanghai 200433, PR of China

e-mail:wxg688@163.com 